\title{
Gentamicin Sulphate Release from a Modified Commercial Acrylic Surgical Radiopaque Bone Cement. I. Influence of the Gentamicin Concentration on the Release Process Mechanism
}

\author{
Eva Díez-Peña, ${ }^{a}$ Gloria Frutos, ${ }^{b}$ Paloma Frutos, ${ }^{c}$ and José Manuel Barrales-Rienda ${ }^{*}, a$ \\ ${ }^{a}$ Departamento de Química-Física de Polímeros, Instituto de Ciencia y Tecnología de Polímeros, Consejo Superior de \\ Investigaciones Científicas; Juan de la Cierva, 3, E-28006 Madrid, Spain: ${ }^{b}$ Departamento de Estadística e Investigación \\ Operativa, Facultad de Farmacia, Universidad Complutense; E-28040 Madrid, Spain: and ${ }^{c}$ Visiting Professor on a \\ sabbatical leave off abscence. Departamento de Farmacia y Tecnología Farmacéutica. Facultad de Farmacia, Universidad \\ Complutense; E-28040 Madrid, Spain. Received March 25, 2002; accepted June 11, 2002
}

The purpose of the present work was the study of the gentamicin sulphate (GS) release from a commercial acrylic bone cement $\mathrm{CMW}-\mathrm{1}^{\circledR}$ with the aims of establishing the influence of the slabs preparation as well as the release mechanism and kinetics. The effect of the amount of GS on the release kinetic parameters has been also investigated. In vitro release studies were performed in a buffered saline solution at pH 7.4 and $37^{\circ} \mathrm{C}$. The GS concentration was determined using an indirect spectrophotometric method with an $o$-phthaldialdehyde as a derivatizing reagent. A commercial and three modified samples were tested. The free and fractured surfaces of the GS cement slabs before and after the release studies were observed by means of scanning electron microscopy (SEM). For low GS concentration loading the release was very incomplete because most of the GS beads were encapsulated by the hydrophobic PMMA matrix. A higher amount of antibiotic was released from cement that has a higher amount incorporated. A model and therefore a mechanism of release based on this model have been proposed. It has allowed us to explain the changes in dissolution kinetics of an acrylic matrix type controlled release system up to $12 \%$ GS loading. The cumulative amount of GS released $M_{t} / M_{i}$, was fitted as a function of time. For lower amounts of GS, the regression analysis $\left(R^{2}>0.99\right)$ revealed that the release is most adequately represented by $M_{t} / M_{i}=b+k t^{n}$, where $b$ represents a burst effect. The goodness of fit decreases as the amount of GS increases. The influence of some other type of release mechanism for higher amounts of GS must be taken into account and a second model for the release, $M_{t} / M_{i}=b+k \cdot[1-\exp (-k t)]$, is proposed. nism

Key words commercial acrylic bone cement; gentamicin sulphate; drug release; modified bone cement; stress cracking; mecha-

Chronic osteomyelitis remains as an important and daunting orthopedic and clinical problem. Conventional treatment using systematic antibiotics is often unsuccessful. High systemic levels of aminoglycoside antibiotics also imply the risk of organ toxicity such as hearing or kidney damages. ${ }^{1)}$

One management method utilizes surgical implantation of antibiotic-impregnated poly(methyl methacrylate) (PMMA) beads into dead space created by osteomyelitic tissue removal, for local delivery of antibiotics. ${ }^{2-4)}$ This method intends to reach high local drug levels while maintaining low systemic levels. Gentamicin sulphate (GS)-PMMA, antibiotic-impregnated or antibiotic-loaded acrylic cements have been used in surgery to prevent or treat osteomyelitis ${ }^{5)}$ since the 1970s. A great number of studies on the amount and rate of GS release from different commercial acrylic bone cements have been published, but an insight into the mechanism and kinetics of the release process is still lacking. ${ }^{6-12)}$ The main aspects on which the present work is based are as follows:

(i) There Exist Some Significant Differences in Behavior among the Different Antibiotic Loaded Acrylic Cement: The antibiotic release from these cements has been the subject of numerous studies. Several factors such as trade name, amount and type of antibiotic incorporated, porosity of the system, its specific area, its surface characteristics ${ }^{13)}$ and some other factors, seem to influence very markedly this release. All these facts have been the reason that the main part of these studies have yield irreconcilable results, which are analyzed with difficulty, and, in some cases, quite selfcontra- dictory. ${ }^{14-16)}$ These apparent discrepancies require explanation. Therefore we tried to perform a reproducible study from which conclusions about the release properties of bone cements and related devices could be extracted. This information may then be used to modify the formulation in order to improve its release characteristics.

(ii) Non Effective Drug Levels: It has been shown in the "in vitro" experiments that much of the antibiotic may be retained within the PMMA matrix, sometimes as much as $90 \%$ of the load. $7,9,11,16)$

(iii) Mechanism and Kinetics for the Precise Control of the Release Process: Due to all the above mentioned, it has not been possible to establish the mechanism and the kinetics of the GS release from commercial acrylic surgical radio opaque bone cement very clearly so far. An essential feature of the acrylic bone cements is shown in experiments carried out in vivo and in vitro by Baker and Greenham, ${ }^{8)}$ who proved that PMMA bone cement that has no defects is impervious to gentamicin. In addition the glass transition of PMMA is far away above room temperature, ${ }^{17)}$ and therefore from the temperature at which the delivery experiments are carried out. From this point of view the diffusion throughout the polymeric matrix seems to be almost unfeasible.

In the present study, special attention has been paid to determine the influence of the amount of the antibiotic incorporated to the bone cement on its release. Different amounts of drug to modify the ratio of antibiotic to polymeric matrix have been added. These modifications will allow us to determine the way in which the antibiotic is released. ${ }^{18)}$ For this 
reason, we have made a systematic analysis on this aspect and therefore we have intended to minimize the influence of some other factors.

\section{Experimental}

Materials Commercial CMW- $1^{\circledR}$ acrylic surgical radio opaque bone cement GS additived was purchased from De Puy Ibérica, S. A. (Madrid, Spain) as a kit of two components, one, a sterile glass ampoule $(18.37 \mathrm{~g})$ containing the liquid component and the other of a sterile package $(40 \mathrm{~g})$ of the powder formulation. Its composition $(\% \mathrm{w} / \mathrm{w})$ is as follows:

(i) The solid component is composed of $4.22 \%$ wt of GS, $84.73 \% \mathrm{wt}$ of poly (methyl methacrylate) (PMMA) beads or microspheres, $1.95 \%$ wt of benzoyl peroxide, which is an initiator of the radical polymerization of monomer from the liquid component and $9.10 \%$ wt barium sulphate used as radio opacifier.

(ii) The liquid component is composed $98.215 \%$ wt of methyl methacrylate (MMA) monomer, $0.816 \% \mathrm{wt}$ of $N, N$-dimethyl- $p$-toluidine (DMPT) which is an activator employed to break down the initiator from the powder component and initiate the polymerization by means of free radicals, $0.945 \%$ wt ethanol, and trace amounts of ascorbic acid $(0.022 \% \mathrm{wt})$ and hydroquinone $(0.002 \% \mathrm{wt})$ which act as inhibitors or stabilizers to prevent premature polymerization of the monomer (MMA).

The following materials were also used as received without any further purification: anhydrous di-sodium hydrogen phosphate, anhydrous potassium hydrogen phosphate, sodium chloride, and sodium borate (Panreac Química S.A. Madrid, Spain); o-phthaldialdehyde and 2-mercaptoethanol (Sigma Aldrich Chemie GmbH, Steinheim, Germany): 2-propanol and methanol (Scharlau-F.E.R.O.S.A., Barcelona, Spain). A commercial gentamicin sulphate with an approximated potency of $604 \mu \mathrm{m} / \mathrm{mg}$ was supplied free of charge by Laboratorios Normon S. A. (Madrid, Spain).

Buffered Reagent Buffered saline solution $\mathrm{pH} 7.4$ was prepared as described in the British Pharmacopoeia (BP A79): anhydrous di-sodium hydrogen phosphate $2.38 \mathrm{~g}$; anhydrous potassium hydrogen phosphate $0.19 \mathrm{~g}$ and sodium chloride $8 \mathrm{~g}$ to $1000 \mathrm{ml}$ with deionized water (Milli-Q)

$o$-Phthaldialdehyde Reagent $o$-Phthaldialdehyde reagent was prepared following a procedure due to Zhang et al. ${ }^{19)}$ It was formulated by adding $2.5 \mathrm{~g}$ of $o$-phthaldialdehyde, $62.5 \mathrm{ml}$ methanol and $3 \mathrm{ml}$ of 2-mercaptoethanol to $560 \mathrm{ml} 0.04 \mathrm{M}$ sodium borate in deionized water solution. The reagent was stored in a brown bottle in the darkness and settled for at least $24 \mathrm{~h}$ prior to use.

Methods. Matrix Plate Preparation Three different procedures to prepare pre-formed cement devices were compared: Hand molding (the method used by medical doctors) and iron and teflon molded samples. For our research, in order to have a well-defined geometry and amount of cement, iron and teflon molded samples had a great advantage to allow a comparison and kinetic studies. But it was necessary to prove that results obtained with molded samples were comparable to those obtained with the hand molded samples. As it will be shown in results section no significant differences among the samples prepared by the three different methods were observed. They were prepared from the two components following the instructions of the cement manufacturing company.

(a) Teflon Mould: Bone cement slabs were prepared following the proto$\mathrm{col}$ as it has been described by Bayston and Milner ${ }^{15)}$ and Wasserlauf et al. ${ }^{20}$ using a teflon mould in a hydraulic press. Specimens were prepared at an initial temperature of $25 \pm 2{ }^{\circ} \mathrm{C}$ and constant humidity $(30 \pm 10 \%)$. Open mixing of powdered and liquid components was performed in a $50 \mathrm{ml}$ beaker. After a careful hand mixing for $2-3$ min with a metal spatula, the cement paste was hand molded. By packing the polymerizing paste into a six cavities Teflon mould that was compressed till the cement had completely hardened, rectangular specimens of $2 \mathrm{~mm}$ thickness, $50.20 \mathrm{~mm}$ length and $25 \mathrm{~mm}$ width were obtained.

(b) Iron Steel Mould: Discs of bone cement were prepared as described by Bayston and Milner ${ }^{15)}$ using an extra sized stainless steel mould (30 $\mathrm{mm} \mathrm{\phi )}$. Thus, $2 \mathrm{~g}$ of the powder mixture component and $1 \mathrm{ml}$ of the commercial liquid component were hand mixed "in situ" to obtain a paste which was submitted to a pressure of $10 \mathrm{~kg} / 1000 \mathrm{psi}$ during $30 \mathrm{~min}$ in a hydraulic press. The discs obtained this way show a very smooth and brilliant surface, with mean dimensions of $30 \mathrm{~mm}$ diameter and $3 \mathrm{~mm}$ thickness.

(c) Hand Molding: The same amounts of the powdered mixture and liquid component were mixed and hand molded till the cement had completely hardened, with an approximate shape to that one of the discs previously obtained. The six hand made discs thus prepared had average dimensions and weights almost similar to those of the disc prepared by compression.
These sequences of operations were carried out to prepare four times concentration GS modified cements. In the powder component a mixture of $40 \mathrm{~g}$ of CMW- ${ }^{\circledR}$ cement free of antibiotic and $6.48 \mathrm{~g}$ of GS was prepared to attain in the modified cement an antibiotic concentration four times higher than the one in the commercial cement. With this mixture the three types of specimens were prepared. In order to determine the effect of the gentamicin sulphate amount on the release process, the Teflon mould procedure was chosen. Specimens with four different gentamicin concentrations were prepared by adding calculated extra amounts of GS to the solid component of the commercial GS additivated CMW- ${ }^{\circledR}$. In this way, commercial GS concentration and four, eight and twelve times commercial GS concentration specimens were obtained. The method of preparation, composition and characteristics of each specimen are gathered in Table 1. In the table "GS added" refers to the spray dried GS added to the bone cement in order to change its GS amount. For Hand and Iron steel molded samples this is the total amount of GS in the sample because a commercial bone cement powder component without gentamicin was used. As it will be show this did not make any significant difference in the results.

Gentamicin Determination An indirect method was required for the spectrophotometric analysis of gentamicin since it does not absorb ultraviolet nor visible light. The $o$-phthaldialdehyde was used as a derivatizing agent. ${ }^{21)}$ It reacts with the amino groups of gentamicin to yield chromophoric products. The $o$-phthaldialdehyde reagent, modified by Zhang et $a l^{19)}$ was prepared as described before. ${ }^{18)}$ The reaction was carried out making $2 \mathrm{ml}$ of our problem gentamicin in solution react with $2 \mathrm{ml}$ of isopropanol (to avoid the precipitation of the products formed) and $2 \mathrm{ml}$ of $o$ phthaldialdehyde reagent. The concentration of gentamicin sulphate was estimated by measuring the absorbance at $332 \mathrm{~nm}$.

The Validation Method Validation of the analytical procedure is required to assure a minimum level of quality in the experimental measurements. The validation of the spectrophotometric method has been done following recommendations given by $\mathrm{ICH}^{22}{ }^{22}$ Some details about the validation of the method employed in this paper have been given elsewhere. ${ }^{23)}$

Scanning Electron Microscopy (SEM) The surface morphological characteristics of the untreated and treated specimens were studied using a Jeol JSM-840 Scanning Electron Microscope. Surfaces and liquid nitrogen fractured surfaces were coated with gold-palladium alloy in a sputter coating apparatus before SEM analysis.

Dissolution Studies in Vitro Release profiles of gentamicin from the cement were measured as follows: one test slab was introduced into a $300 \mathrm{ml}$ Pyrex glass reactor containing $250 \mathrm{ml}$ of the phosphate buffered saline solution $(\mathrm{pH}=7.4)$ with a water circulating jacket. ${ }^{23)}$ The test solution was maintained at $37{ }^{\circ} \mathrm{C}$ and mechanically stirred at $150 \mathrm{rpm}$. Aliquots amounts $(3 \mathrm{ml})$ of the solution were withdrawn at suitable time intervals, passed through a $0.45 \mu \mathrm{m}$ millipore membrane filter, and diluted with the buffered reagent, when necessary, for measurements in the spectrometer. Four slabs were tested for each sample show in Table 1.

\section{Experimental Results and Discussion}

The experimental results for the time-dependent release of GS are best explained in terms of the setting processes and structure of the acrylic bone cement. It may be interesting and appropriate to give some thought to the possible mechanisms of GS release and the manner in which it might be modeled. The release mechanism must necessarily be complex, due to the fact that the cement has a heterogeneous and complex structure. The cement is the product of a reaction between a powdered mixture constituted fundamentally by PMMA beads, GS sulphate beads and barium sulphate particles and a liquid composed almost completely of MMA monomer. After mixing and polymerization of both components, the solid particles of PMMA are dispersed in a PMMA solid matrix which encloses them generating a heterogeneous morphology. PMMA is present both in the matrix and in the beads. Even in simple heterogeneous macromolecular structures diffusion processes seem to be very complicated. ${ }^{24)}$ Diffusion in amorphous polymers is only possible above its glass transition temperature. We have, on one hand, that the migrant (GS) is a drug in its salt form of considerable molec- 
Table 1. Mean Composition and Characteristics of the Surgical CMW- $1^{\circledR}$ Gentamicin Sulphate Radiopaque Bone Cement and of Their Modified Analogous

\begin{tabular}{|c|c|c|c|c|c|c|c|c|}
\hline & \multirow{2}{*}{ Sample } & \multirow{2}{*}{$\begin{array}{c}\text { Unmodified commercial } \\
\text { liquid } \\
\text { component } \\
(\% \mathrm{wt})\end{array}$} & \multicolumn{2}{|c|}{$\begin{array}{l}\text { Composition modified } \\
\text { powder component }\end{array}$} & \multirow{2}{*}{$\begin{array}{l}\text { Gentamicin content } \\
\text { (Polymerized cement) } \\
\text { (\%wt) }\end{array}$} & \multicolumn{3}{|c|}{ Slab characteristics } \\
\hline & & & $\begin{array}{l}\text { Unmodified } \\
\text { powder } \\
(\% \mathrm{wt})\end{array}$ & $\begin{array}{l}\text { GS } \\
\text { added } \\
(\% w t)\end{array}$ & & $\begin{array}{l}\text { Weight } \\
(\mathrm{g})\end{array}$ & $\begin{array}{l}\text { Area } \\
\left(\mathrm{cm}^{2}\right)\end{array}$ & $\begin{array}{l}\text { Thickness } \\
(\mathrm{cm})\end{array}$ \\
\hline $1 \mathrm{GS}$ & (Teflon mould) & 32.88 & 67.12 & - & 2.89 & 3.870 & 30.32 & 0.29 \\
\hline \multirow[t]{2}{*}{$4 \mathrm{GS}$} & $\begin{array}{l}\text { (Hand molding) } \\
\text { (Teflon mould) }\end{array}$ & $\begin{array}{l}33.33 \\
28.91\end{array}$ & $\begin{array}{l}57.37 \\
63.04\end{array}$ & $\begin{array}{l}9.29 \\
8.05\end{array}$ & $\begin{array}{r}9.29 \\
10.73\end{array}$ & $\begin{array}{l}2.762 \\
2.815\end{array}$ & $\begin{array}{l}18.83 \\
28.85\end{array}$ & $\begin{array}{l}0.39 \\
0.18\end{array}$ \\
\hline & (Iron steel mould) & 33.33 & 57.37 & 9.29 & 9.29 & 2.898 & 18.34 & 0.30 \\
\hline $8 \mathrm{GS}$ & (Teflon mould) & 26.55 & 56.71 & 16.74 & 19.14 & 2.795 & 28.12 & 0.21 \\
\hline $12 \mathrm{GS}$ & (Teflon mould) & 25.36 & 50.98 & 23.66 & 25.81 & 4.035 & 29.63 & 0.27 \\
\hline
\end{tabular}

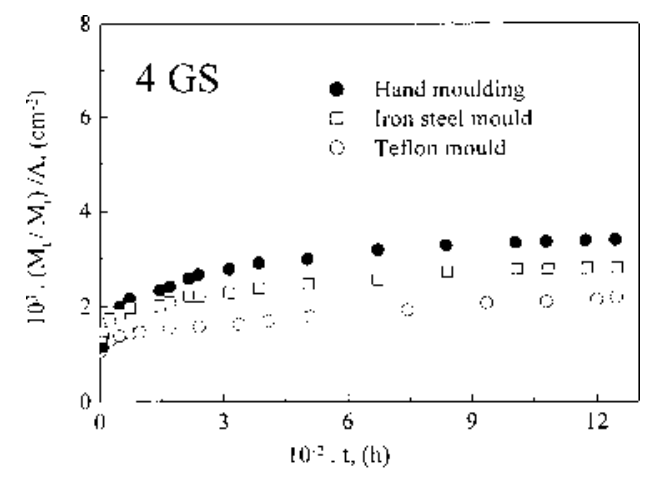

Fig. 1. Influence of the Mode of Slabs Preparation Expressed as the Experimental Cumulative Mean Amount of Gentamicin Sulphate Released Normalized per Unit of Exposed Area $\left(M_{t} / M_{i}\right) / A\left(\mathrm{~cm}^{-2}\right)$ as a Function of Elution Time $t$, (h) for a Series of Modified Commercial Surgical Acrylic Drug Delivery Bone Cement (4 GS)

For mode of slabs preparation, composition and GS content of the samples see Table 1 .

ular weight and volume that is soluble in water and on the other hand we have a polymeric matrix fundamentally constituted by a hydrophobic polymer. From this point of view it seems to be logic that the polymeric matrix should be impermeable to GS.

Figures 1 and 2 compare the release profiles obtained with the different samples assayed. Figure 1 shows the results for series of samples with the same amount of GS incorporated (4 GS), but prepared using different procedures, i.e.: (a) Teflon mould, (b) iron steel mould, and (c) hand molded samples. The cumulative percentage of drug released $\left(M_{t} / M_{i}\right)$ was normalized by the sample surface $\left(A, \mathrm{~cm}^{2}\right)$ in order to minimize differences due to the sample morphology. In spite of this normalization only slight differences can be noticed among the samples. No significant differences among the samples prepared by the three different methods were observed. The differences found when the amount of GS is modified (as is shown in Fig. 2) are much bigger compared to the differences due to the method of preparation which were not significative and will not be taken into account in the discussion of the results. Therefore, we will only consider hereafter samples prepared by one of the methods, namely, method (a).

In Fig. 2 can be noticed the differences in the amount of GS incorporated to the cement give place to great changes in

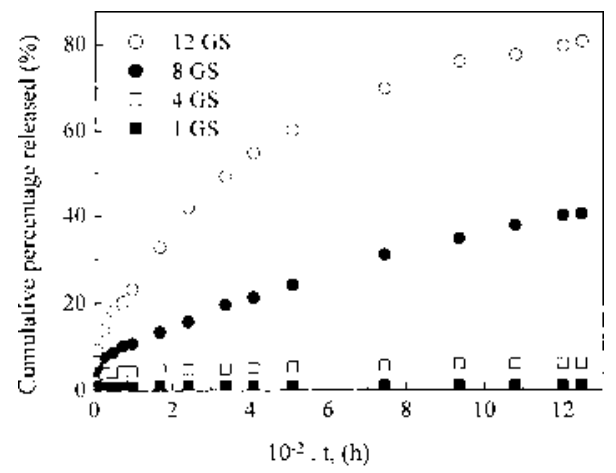

Fig. 2. Experimental Cumulative Mean Amount of Gentamicin Sulphate Elution Profile Normalized per Unit Exposed Area $\left(M_{t} / M_{i}\right) / A\left(\mathrm{~cm}^{-2}\right)$ as a Function of Elution Time $t$, (h) for a Series of Unmodified and Modified Commercial Surgical Acrylic Drug Delivery Bone Cement (1 GS, 4 GS, 8 GS and 12 GS)

For composition and GS content of the samples see Table 1.

the cumulative percentage of drug released from the cement. In this case a normalization of results was not necessary because of the similar morphology of samples prepared using the same procedure.

Figure 3a shows a SEM microphotograph of the free surface of GS beads. It reveals a regular structure of sphericalshaped particles differing in size from 2 to $45 \mu \mathrm{m}$ and a smooth surface. Figure $3 \mathrm{~b}$ shows a SEM microphotograph of the free surface of PMMA beads of the commercial bone cement. They present oval and spherical particles with a rugose surface texture. Therefore both types of particles are quite different from the point of view of their respective morphologies.

Figures $4 \mathrm{a}$ and $\mathrm{b}$ show microphotographs of fracture surfaces of untreated samples 4 GS and 12 GS. Because of the GS incompatibility due to its hydrophilic character, GS beads appear as a disperse heterogeneous phase in the PMMA matrix. On the contrary PMMA beads are not easily distinguishable from the PMMA matrix because both are compatible. Both effects are easily distinguishable if we compare samples $4 \mathrm{GS}$ (GS content $=10.73 \% \mathrm{wt}$ ) and $12 \mathrm{GS}$ (GS content $=$ $25.81 \% \mathrm{wt}$ ). Thus the texture of the polymeric matrix and PMMA beads does not change but the traces due to GS beads are clearly increased in 12 GS sample as it can be expected.

Figures $5 \mathrm{a}$ and $\mathrm{b}$ show microphotographs of the external 

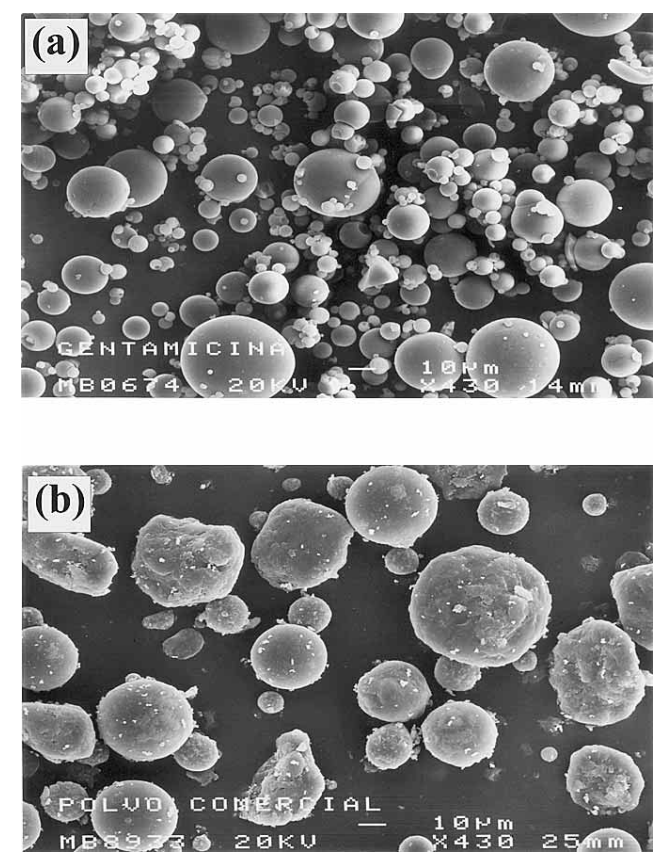

Fig. 3. (a) SEM Showing the External Surface of Gentamicin Sulphate Beads and (b) SEM Showing the External Surface of Poly(methyl methacrylate) Beads from the CWM-1 Acrylic Bone Cement Solid Component
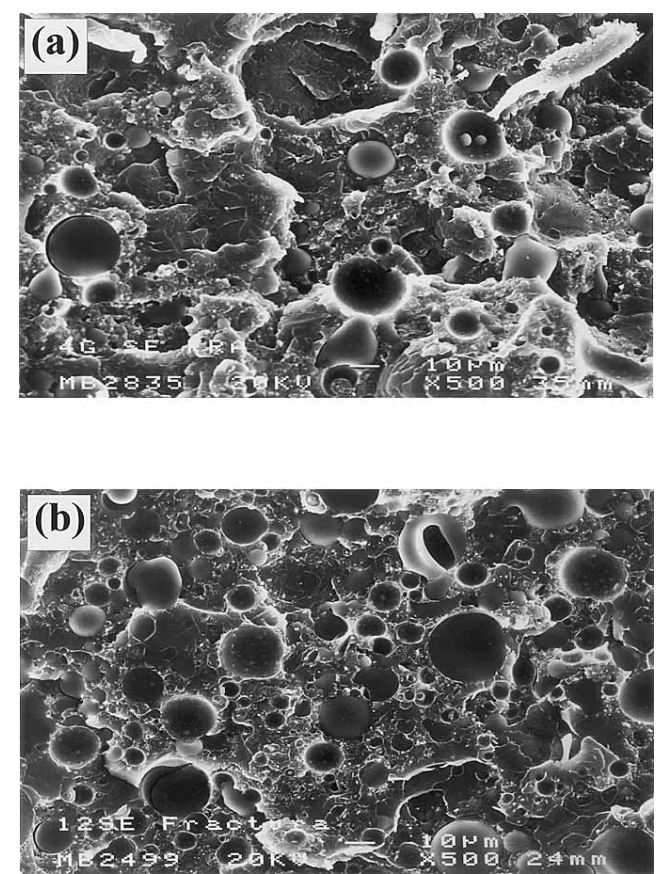

Fig. 4. SEMs Showing the Fractured Surface of Two Unsoaked and Modified Commercial CWM-1 Acrylic Bone Cements before the Release Assays

(a) (Sample 4 GS ); and (b) (Sample 12 GS). For mode of slabs preparation, composition and GS content of the samples see Table 1 .

surface of two samples of unmodified 1 GS (GS content $=$ $2.89 \% \mathrm{wt}$ ) and modified $12 \mathrm{GS}$ (GS content $=25.81 \% \mathrm{wt}$ ) bone cements after $1250 \mathrm{~h}$ of release test. In the surfaces of the specimens there are a large number of voids. We may assume, that this may be due to gentamicin beads located near to the external surface that have been removed by a dissolution process leaving a porous structure. The commercial cement surface of Fig. 5a shows some very thick cracks but it
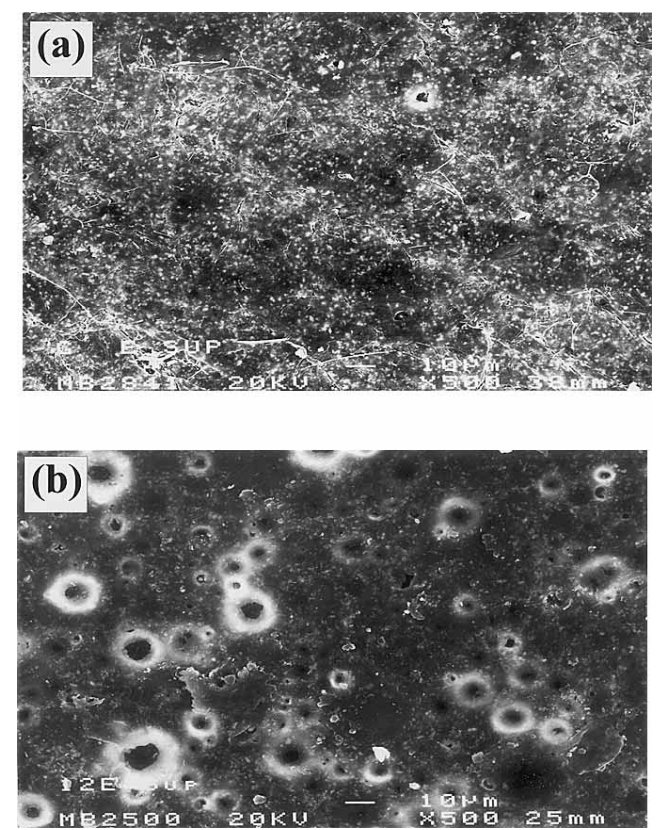

Fig. 5. SEMs Showing the External Surface (a) of an Unmodified Commercial CWM-1 Acrylic Bone Cement (Sample 1 GS) and (b) of a Modified CWM-1 Acrylic Bone Cement (Sample 12 GS) after Having Been in the Buffer Solution at $37^{\circ} \mathrm{C}$ for $1250 \mathrm{~h}$

For mode of slabs preparation, composition and GS content of the samples see Table 1

does not shown neither apparent signs of craters nor polygonal forms. For a higher loaded GS sample, Fig. 5b, there are a lot of craters which have been created by the extraction GS from the more external GS beads covered by a thin brittle PMMA layer. Some of these craters seem to be the remains of the GS beads. The same effect has been observed by Fukazawa et al. ${ }^{25,26)}$ in the case of the erosion of glassionomer cements. The buffer would dissolve these GS beads gradually leaving a number of pores near the surface of the GS modified cement slabs.

Figures 6 and 7 show the microphotographs of the fractured surfaces of bone cement slabs after immersion for $1250 \mathrm{~h}$ of a series of four samples differing in GS concentration. All have been taken at the same magnification and, show the different pore widths and the structural differences among the surfaces of the four samples after the release tests for the same period of time. From these Figs. 6 and 7, we can conclude that after MMA polymerization and before the releasing experiment, namely before the sample contacts the buffer, the GS beads are not free but embedded and covered by the PMMA matrix, which also encloses the PMMA beads forming part of it as a continuous phase.

The surface morphology of the soaked as well as the unsoaked gentamicin-loaded cement revealed a considerable amount of voids or craters and cracks. The SEM micrographs also showed bubble-like voids and cracks in the cement that may have been utilized as conduits for antibiotic release. After the curing of the bone cement its external morphology macroscopically resembles the external surface of a hazel-nut chocolate table where the gentamicin sulphate beads may be represented by the nuts. We have a polymeric matrix where the PMMA beads have been left embedded forming a quasi homogeneous phase with the matrix and then completely dis- 

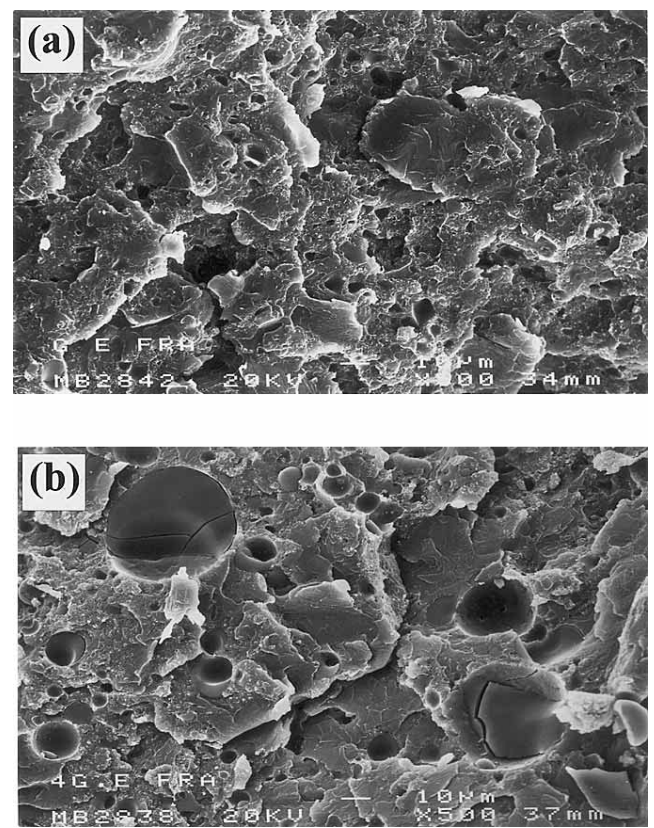

Fig. 6. SEMs Showing the Fractured Surface of an Unmodified and a Modified Commercial CWM-1 Acrylic Bone Cements after Having Been in the Buffer Solution at $37^{\circ} \mathrm{C}$ for $1250 \mathrm{~h}$

(a) (Sample $1 \mathrm{GS}$ ); and (b) (Sample $4 \mathrm{GS}$ ). For mode of slabs preparation, composition and GS content of the samples see Table 1.
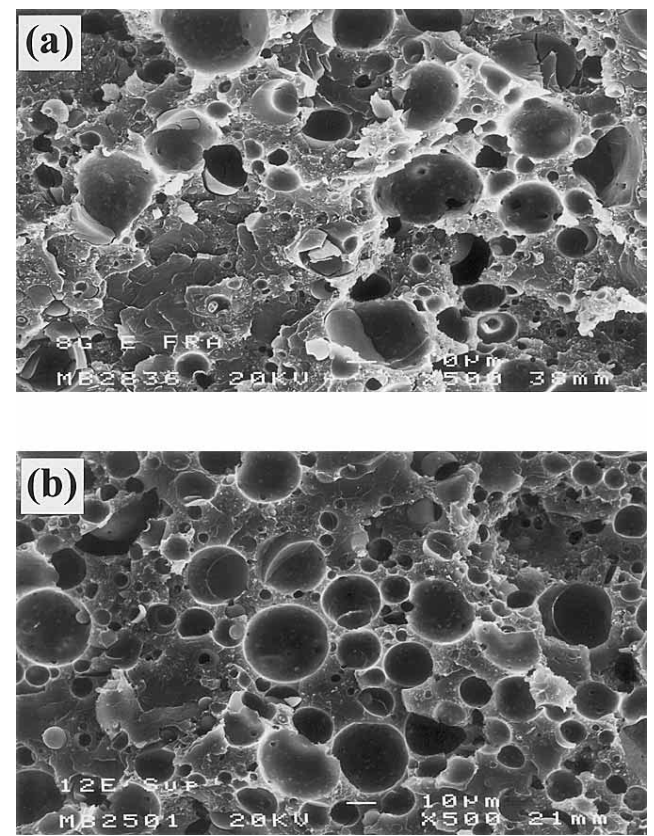

Fig. 7. SEMs Showing the Fractured Surface of Two Modified Commercial CWM-1 Acrylic Bone Cements after Having Been in the Buffer Solution at $37^{\circ} \mathrm{C}$ for $1250 \mathrm{~h}$

(a) (Sample 8 GS); and (b) (Sample 12 GS). For mode of slabs preparation, composition and GS content of the samples see Table 1.

persed GS beads and the radio opacifier particles.

To describe the drug release Lindner and Lippold ${ }^{27)}$ proposed and expanded Korsmeyer et al. ${ }^{28)}$ equation adding a constant term $b$, to take into account the "burst effect",

$$
M_{t} / M_{i}=b+k t^{n}
$$

From the mathematical point of view, the intercept $b$ is

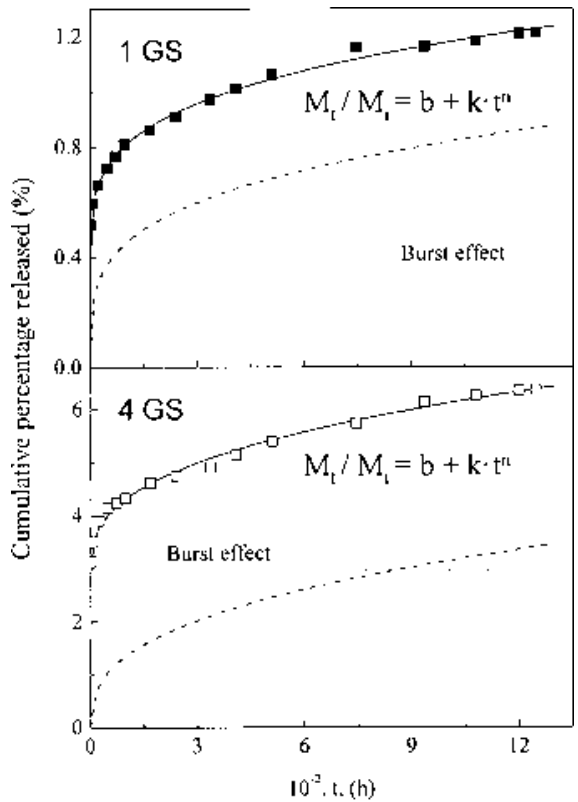

Fig. 8. Experimental and Calculated Cumulative Percentage (\%) of Gentamicin Sulphate Released as a Function of Time $t$, (h) According to Eq. 1 (Full Line) of a Commercial and Modified Acrylic Bone Cement Drug Delivery Devices Bone Cement (Sample 1 GS and 4 GS)

Slabs have been made by mould compression in a home made teflon mould.

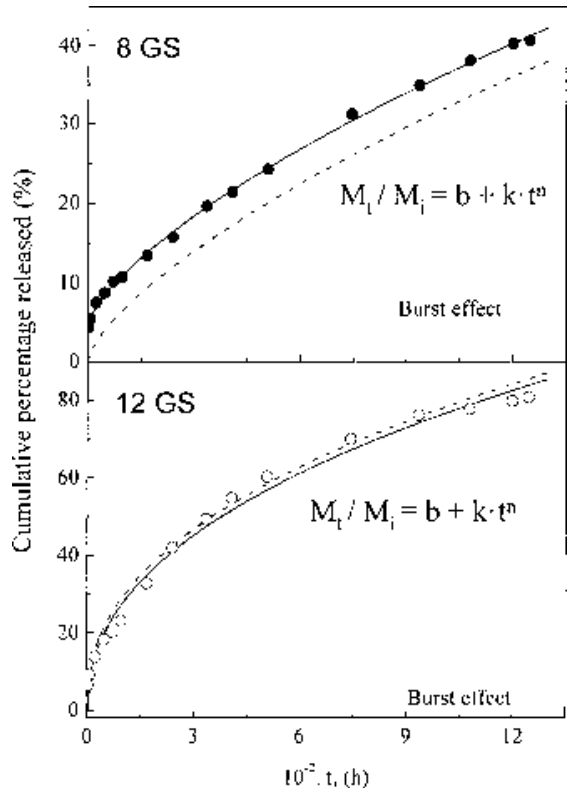

Fig. 9. Experimental and Calculated Cumulative Percentage (\%) of Gentamicin Sulphate Released as a Function of Time $t$, (h) According to Eq. 1 (Full Line) of Two Modified Acrylic Bone Cement Drug Delivery Devices Bone Cement (Samples 8 GS and 12 GS)

Slabs have been made by mould compression in a home made teflon mould

very important, because its disregard leads to a poorer correlation of the experimental data with the calculated curves. However, from the mechanistic point of view, as would be mentioned later on, it represents and characterizes the known "burst effect". When a soluble drug dissolves rapidly from the surface creating a "burst effect", some of the drug in the surface will desorbed at an initially high rate. Therefore Eq. 1 may be appropriate to analyze and interpret release data.

Figures 8 and 9 show the patterns of gentamicin sulphate 
Table 2. Statistical Parameters and Standard Errors According to Eq. 1

\begin{tabular}{rrrrr}
\hline \hline \multirow{2}{*}{ Sample } & \multicolumn{4}{c}{$M_{t} / M_{i}=b+k t^{n}$} \\
\cline { 2 - 5 } & \multicolumn{1}{c}{$b$} & $k$ & $n$ & $R^{2}$ \\
\hline 1 GS & 0.357 & 0.136 & 0.260 & 0.9960 \\
4 GS & 2.966 & 0.254 & 0.364 & 0.9919 \\
8 GS & 4.445 & 0.288 & 0.681 & 0.9988 \\
12 GS & -1.539 & 4.136 & 0.425 & 0.9909 \\
\hline
\end{tabular}

release from the bone cement in a phosphate buffer at $\mathrm{pH}=7.4$ at $37^{\circ} \mathrm{C}$. As it also shown in these Figs. 8 and 9 the amount of GS released from the cements into the buffer solution were fitted by Eq. 1. Values of the parameters and correlation coefficients are given in Table 2.

The increase of the $n$ value, above 0.5 for the 8 GS sample, and the decrease of the goodness of fit for the 12 GS sample as can be seen in Fig. 9 may be indicating a change in the release mechanism, for high GS concentration samples. Therefore, we propose a second model to be applied in this concentration region

A second model to describe the release mechanism was fitted against the experimental data,

$$
M_{t} / M_{i}=b+k \cdot[1-\exp (-n t)]
$$

The term $k \cdot[1-\exp (-n t)]$ is associated with a Noyes and Whitney dissolution process. ${ }^{29,30)}$ The statistical evaluation is shown in Figs. 10 and 11 and the parameters are given in Table 3. The goodness of the fit for low GS concentration samples (1 GS and 4 GS) is much worse than with the previous model. However, for the high concentration samples the goodness of the fit is very good. For these samples this analysis has a more realistic meaning as can be concluded from the values of $b$ (burst effect). As it can be seen in Table 2, in Eq. 1 fit for sample $12 \mathrm{GS}$, it was necessary to give $b$ a negative values, and the physical explanation of this fact is not very clear. On the contrary, the value of $b$ for Eq. 2 fit is positive, as expected, and also shows an increase from 8 GS to 12 GS, which has a proper physical explanation due to the higher amount of GS incorporated into the sample.

As an explanation for the fracture surface morphology and experimental release results data two mechanisms have been proposed. For low GS concentration samples most of the drug remain isolated by the polymeric matrix and therefore can not be released at all. The defective drug release is due to imperfection of the polymeric covering on the most external layers of the sample, or stress cracking of the thin layer of PMMA covering the most external GS beads. This fact allows the buffered saline solution to penetrate the polymeric matrix, dissolving the drug located close to the external layers of the sample and releasing it. This effect is increased by increasing the drug concentration in the sample from $2 \mathrm{GS}$ to 4GS, leading to higher amount of imperfections of the PMMA covering and a higher amount of drug on the most external layers of the sample. Nevertheless most of the drug loading still remains isolated in the inner layers of the sample. On the contrary, when the amount of GS incorporated is increased from 4 GS to 8 GS a threshold seems to be reached where the release mechanism is changed and some new effects must be taken into account. The spatial proximity of the GS beads as shown in the SEM photographs, may allow the

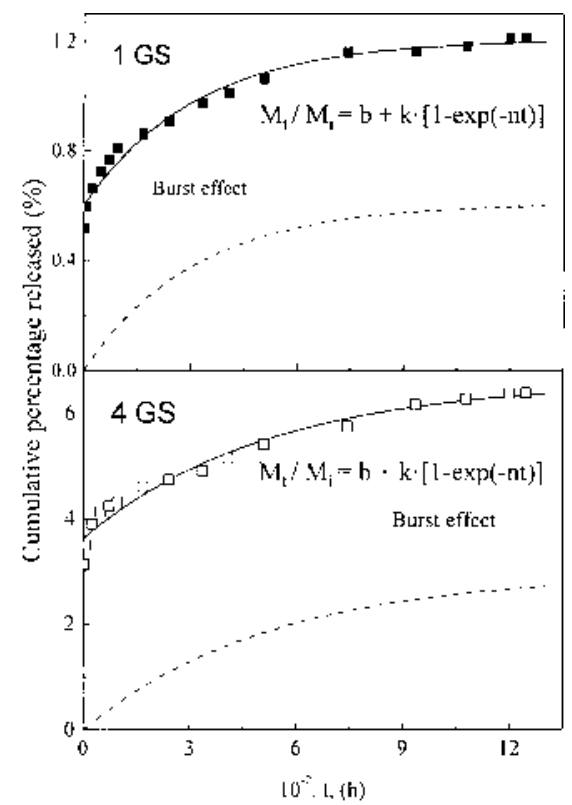

Fig. 10. Experimental and Calculated Cumulative Percentage (\%) of Gentamicin Sulphate Released as a Function of Time $t$, (h) According to Eq. 2 (Full Line) of a Commercial and a Modified Acrylic Bone Cement Devices (Samples 1 GS and 4 GS)

Slabs have been made by mould compression in a home made teflon mould.

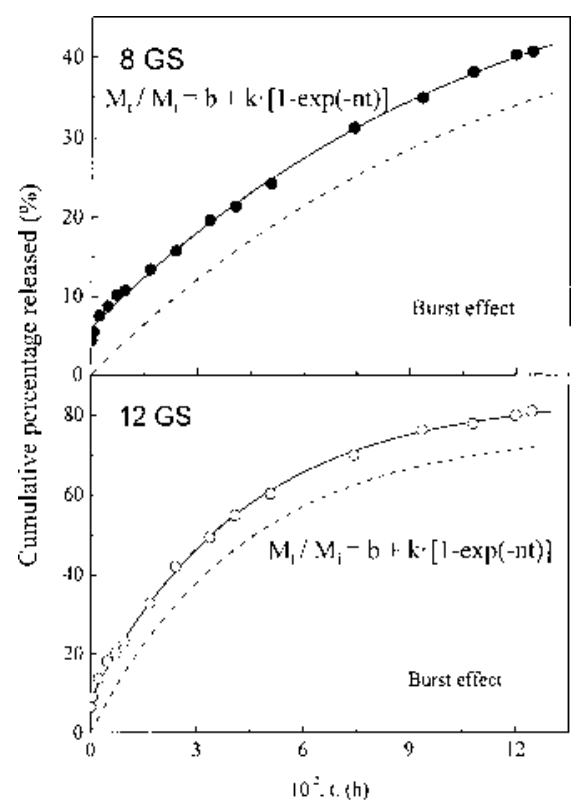

Fig. 11. Experimental and Calculated Cumulative Percentage (\%) of Gentamicin Sulphate Released as a Function of Time $t$, (h) According to Eq. 2 (Full Line) of Two Modified Commercial Acrylic Bone Cement Drug Delivery Devices (Samples 8 GS and 12 GS)

Slabs have been made by molding compression in an home made teflon mould.

Table 3. Statistical Parameters and Standard Errors According to Eq. 2

\begin{tabular}{rcccc}
\hline \hline \multirow{2}{*}{ Sample } & \multicolumn{4}{c}{$M_{t} / M_{i}=b+k \cdot[1-\exp (-n t)]$} \\
\cline { 2 - 5 } & $b$ & $k$ & $n$ & $R^{2}$ \\
\hline 1 GS & 0.601 & 0.606 & 0.003 & 0.9788 \\
$4 \mathrm{GS}$ & 3.634 & 2.962 & 0.002 & 0.9660 \\
$8 \mathrm{GS}$ & 5.934 & 51.795 & 0.001 & 0.9976 \\
$12 \mathrm{GS}$ & 8.510 & 75.932 & 0.002 & 0.9988 \\
\hline
\end{tabular}



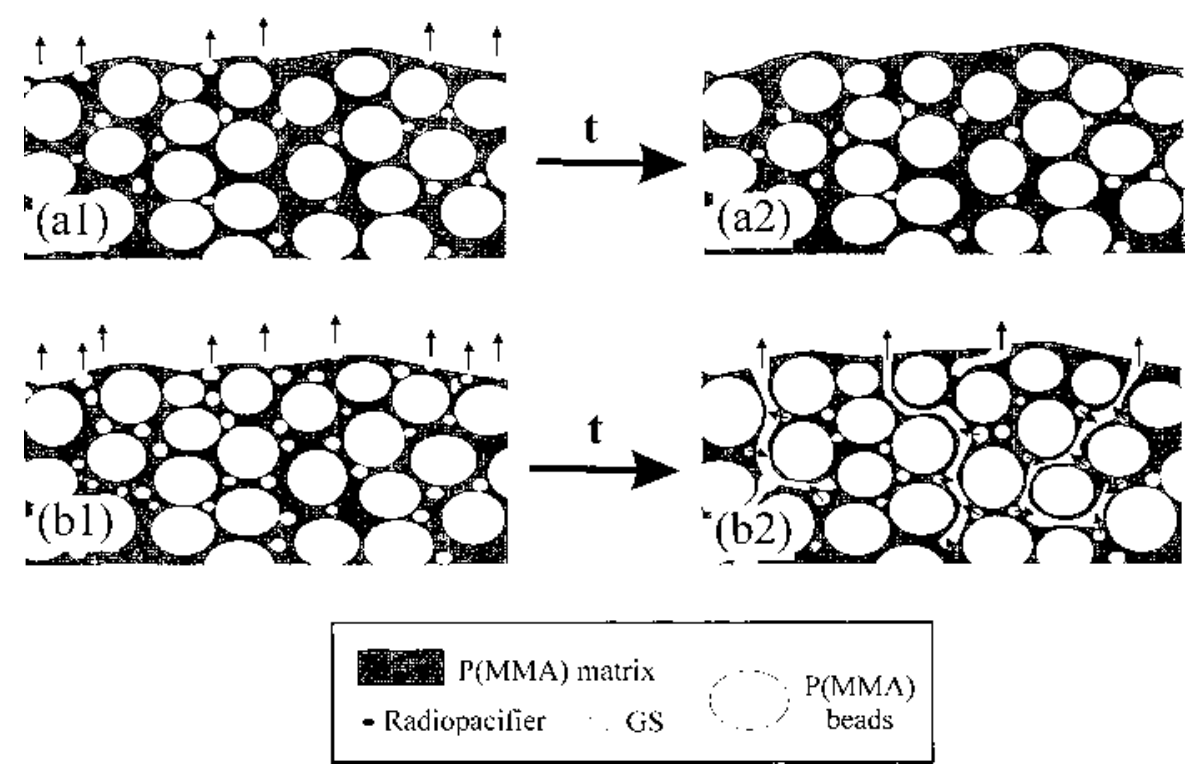

Fig. 12. Scheme of the Proposed Change in the Release Mechanism (a) Low GS Amount Samples, below the Threshold (1 GS, 4 GS) and (b) High GS Amount Samples, above the Threshold (8GS, 12 GS)

access of the soaking medium to the inner layers of the sample (stress cracking or pre-existent imperfections). This leads to the creation of some elution paths which may allow the complete release of the drug contained in the sample. The proposed processes that take place below and above the threshold are shown in Fig. 12. This is corroborated by the abrupt increase in the amount of drug released by $8 \mathrm{GS}$ and 12 GS concentration samples and the change in the fitting parameters as shown before.

\section{Conclusions}

For the commercial GS concentration loading (2.89 wt \%) acrylic bone cement, namely, the standard commercial cement $\left(\mathrm{CMW}-\mathrm{1}^{\circledR}\right)$, the GS release was incomplete. This is due to the fact that most of the drug was encapsulated by the polymeric PMMA matrix. This hydrophobic polymer is impervious to GS, and the release is mainly due to imperfections of the polymeric covering of the most external layers of the sample.

The effect of the addition of extra amounts of GS to this standard surgical CMW- ${ }^{\circledR}$ radio opaque bone cement used in the preparation of the slabs on the kinetic parameters as well as the influence of the method employed to prepare the slabs have been also investigated. The amount of drug released can be only very slightly modified by the method of preparation.

On the contrary, the amount of GS seems to have a dramatic effect. For low GS concentration modified sample $(9.29-10.73 \% \mathrm{wt})$ the release is also mainly controlled by the imperfections of the matrix.

For higher amounts of GS incorporated into the sample $(19.14-25.81 \% \mathrm{wt})$ the release mechanism is changed. An abrupt increase in the amount of drug released takes place, that allows the almost complete release of the drug incorporated. The release experimental results have been supported from the data furnished by SEM. According to the SEM microphotographs, the release may take place by means of a two consecutive or simultaneous solution-diffusion processes through cracks, voids or in general through morphological defects in the matrix and new created cracks produced from frozen stresses in active media of the polymeric material covering gentamicin sulphate beads i.e. a irregular thick shells of PMMA covering GS beads.

The results obtained in this experiments show that the amount of GS incorporated to the bone cement has a dramatic effect on the release mechanism of the drug. Therefore, the relative release rate can be modified by the amount of drug incorporated. This fact, may have a great interest for some commercial devices, intended for a local release of antibiotics in orthopedic surgery, in which the release of drug has a major importance above any further considerations, for instance, mechanical properties. For a better knowledge, the impact on the mechanical properties of the system is currently under research in our group. In addition this results are also quite useful to rationalize changes in the bone cement composition in order to improve its drug release properties.

During the "in vitro" release experiment measurements what we are really measuring are changes of the amount of drug appearing in the buffer solution as a function of time. At all events, it would represent the overall amount of GS which leaves the external surface of the sample. This process is the only one which may be modeled by the kinetic equations. It is usually done by the best fitting of adjustable parameters. Really, we have not any direct information on what is taking place inside the sample. Despite the advances in the study in vitro of drug delivery by homogeneous systems, gels, solid mixtures, etc., the delivery of antibiotics from bone cements remains a considerable problem.

Acknowledgements This work was supported in part by a grant from the Comisión Asesora de Investigación Científica y Técnica de la Subdirección General de Promoción de la Investigación from the Ministerio de Educación y Ciencia PB95-0134-C02-01 and by a grant from the Dirección General de Investigación de la Consejería de Educación y Cultura de la Comunidad Autónoma de Madrid 07M/0069/1998. The authors would like to extend their gratitude to $\mathrm{Dr}^{\mathrm{a}}$. Maria Isabel Estrella Pedrola of the Instituto de Fermentaciones Industriales for providing facilities for using the U-VI spectrophotometer. No benefits in any form have been received or will be re- 
ceived from a commercial party related directly or indirectly to the subject of this article.

\section{References}

1) Schmidt C., Wenz R., Nies B., Moll F., J. Controlled Release, 37, 8394 (1995).

2) Cierny G., Mader J. T., Orthopedics, 7, 1557-1564 (1984).

3) Cierny G., Mader J. T., Pennick J. J., Contemp. Orthop., 10, 17-37 (1985).

4) Mader J. T., Calhoun J., Cobos J., Antimicrob. Agents Chemother, 41, 415-418 (1997).

5) Trippel S. B., Bone Joint Surg. [Am], 68A, 1297-1302 (1988).

6) Buchholz H. W., Elson R. A., Heinert K., Clin. Orthop., 190, 96-108 (1984).

7) Goodell J. A., Flick A. B., Hebert J. C., Howe J. G., Am. J. Hosp. Pharm., 43, 1454-1461 (1986).

8) Baker A. S., Greenham L. W., J. Bone Joint Surg. [Am], 70, 15511557 (1988).

9) Calhoun J. H., Mader J. T., Am. J. Surg., 157, $443-449$ (1989).

10) Stabile D. E., Jacobs A. M., J. Am. Pediat. Med. Ass., 80, 354-359 (1990).

11) Miclau T., Dahners L. E., Lindsey R. W., J. Orthop. Res., 11, 627632 (1993).

12) Meyer J. D., Falk R. F., Kelly R. M., Shively J. E., Withrow S. J., Dernell W. S., Kroll D. J., Randolph T. W., Manning M. C., J. Pharm. Sci., 87, 1149-1154 (1998).

13) Masri B. A., Duncan C. P., Beauchamp C. P., Paris N. J., Arntorp J., J. Arthroplasty, 10, 453-459 (1995).

14) Wahlig H., Dingeldein E., Acta Orthop. Scandinavica, 51, 49-56
(1980).

15) Bayston R., Milner R. D. G., J. Bone Joint Surg. [Br], 64B, 460—464 (1981).

16) Levin P. D., J. Bone Joint Surg. [Br]. 57-B, 234-237 (1975).

17) Fox T. G., Goode W. E., Gratch S., Huggett C. M., Kincaid J. F., Spell A., Stroupe J. D., J. Polym. Sci., 31, 173-177 (1958).

18) Frutos P., Diez-Peña E., Frutos G., Barrales-Rienda J. M., Biomaterials, 23, 3787-3797 (2002).

19) Zhang X., Wyss U. P., Pichora D., Goosen M. F. A., J. Pharm. Pharmacol., 46, 718-724 (1994).

20) Wasserlauf S., Warshawsky A., Arad-Yelin R., Mazur Y., Salama R., Dekel S., Bull. Hosp. Joint Dis., 53, 68-74 (1993).

21) Sampath S. S., Robinson D. S., J. Pharm. Sci., 79, 428-431 (1990).

22) International Conference on Harmonization. The European Agency for the Evaluation of Medical Products. ICH Topic Q 2B. Note for Guidance on Validation of Analytical Procedures: Methodology (1996).

23) Frutos-Cabanillas P., Diez-Peña E., Barrales-Rienda J. M., Frutos G., Int. J. Pharmaceut., 209, 15-26 (2000).

24) Koros W. J. (ed.), "Barrier Polymers and Structures," 423 ACS Symposium Series, American Chemical Society, Washington, 1990.

25) Fukazawa M., Matsuya S., Yamane M., J. Dent. Res., 66, 1770-1774 (1987).

26) Fukazawa M., Matsuya S., Yamane M., J. Dent. Res., 69, 1175-1179 (1990).

27) Lidner W. D., Lippold B. C., Pharm. Res., 12, 1781-1785 (1995).

28) Korsmeyer R. W., Gurny R., Doelker E., Buri P., Peppas N. A., Int. J. Pharmaceut., 15, 25-35 (1983).

29) Noyes A. A., Whitney W. R., Z. Physik. Chem., 23, 689-692 (1897).

30) Noyes A. A., Whitney W. R., J. Am. Chem. Soc., 19, 930-934 (1897). 